\title{
The role of platinum-based therapy for HER-2-positive breast cancer
}

\author{
A. A. Joy, J. R. Mackey \\ Department of Medical Oncology, Cross Cancer Institute, Edmonton, Alberta, Canada.
}

\begin{abstract}
Trastuzumab is a monoclonal antibody targeting the human epidermal growth factor receptor-2 (HER-2) protein, the mediator of an aggressive breast cancer phenotype. Trastuzumab has an important role in the treatment of metastatic breast cancer (MBC) where it improves multiple endpoints, including overall survival. More recently, striking activity is also seen in non-MBC, with substantial benefits seen in both the neoadjuvant and adjuvant settings. Experimental data has identified synergistic therapeutic effects of platinum containing chemotherapeutic regimens when combined with trastuzumab. We review the pre-clinical and rapidly evolving clinical evidence supporting platinum and trastuzumab combinations for HER-2-positive breast cancer.
\end{abstract}

Keywords: Breast cancer; HER-2; Platinum-based chemotherapy; Trastuzumab

\section{Introduction}

Breast cancer is the most frequently diagnosed malignancy in women worldwide accounting for $23 \%$ of all cancer cases in women, with 1.15 million worldwide cases in 2002 alone [1]. Although improvements in breast cancer detection and treatment contribute to declining breast cancer-specific mortality rates over the last decade [2], the efficacy of standard systemic therapy is suboptimal; women still experience relapses despite state-of-the-art adjuvant chemotherapy and hormonal therapy, and distant metastatic disease most frequently leads to premature death. Combining traditional chemotherapy with novel, molecularly targeted therapeutics, however, is showing particular promise.

Correspondence to: John R. Mackey, MD, Department of Medical Oncology, Cross Cancer Institute, 11560 University Avenue, Edmonton, AB, Canada, TG6 1Z2. E-mail: johnmack@cancerboard.ab.ca; Tel: +1 780 432 8221; Fax: +1 7804328888

Received: 14/09/05

Revised: 08/12/05

Accepted: 06/10/05

First published online 17/02/06

$\mathrm{BCO} / 471 / 2005 / \mathrm{FO}$

\section{Human epidermal growth factor receptor-2}

The growing understanding the molecular biology of breast cancer has identified new targets for therapy. We now appreciate that growth factor receptors can trigger intracellular signaling cascades that modulate the cell cycle and apoptosis, and thereby influence the behavior of breast cancer cells. The transmembrane receptor, human epidermal growth factor receptor-2 (HER-2) contains a unique extracellular binding domain, and possesses intrinsic intracellular tyrosine kinase activity. Although HER-2 does not appear to have a single-specific ligand [3], it contributes to signaling via formation of homo/heterodimers with members of the epidermal growth factor receptor family (EGFR). Amplification of the HER-2 gene leads to cell surface over-expression of the protein, enhances dimerization of HER-2, thereby triggering intracellular tyrosine kinase activity and signal transduction pathways. The ultimate result of this HER-2-driven signaling is the promotion of cell growth, division, metastasis, and angiogenesis, and the inhibition of apoptosis.

HER-2 is over-expressed or amplified in $20-30 \%$ of human breast tumors [3,4]. Over-expression has been 
associated with negative clinical prognostic factors such as high tumor grade, DNA aneuploidy, high cell proliferation rate, estrogen/progesterone negativity, and p53 mutation. Aberrations in a variety of other molecular biomarkers of breast cancer invasiveness and metastasis have also been observed [5-7]. As a result, breast cancers with HER-2 over-expression have been consistently associated with a more aggressive disease course and poorer overall prognosis when compared to non-HER-2 over-expressing breast cancer.

\section{Trastuzumab}

Trastuzumab (Herceptin ${ }^{\circledR}$ ) is a humanized monoclonal immunoglobulin G1 (IgG1) antibody that specifically targets the extracellular domain (ECD) of HER-2. Its mechanisms of action include activation of antibody-dependent cellular cytotoxicity, direct disruption of receptor dimerization and signaling of downstream effectors, receptor internalization/degradation, and inhibition of proteolytic cleavage of the HER-2 ECD. The therapeutic effect in model systems is reduced cellular proliferation, suppression of angiogenesis, and ultimately cell cycle arrest [8].

Breast cancers with the HER-2 alteration can be identified by protein over-expression, as assessed by immunohistochemistry (IHC), and/or gene amplification, as assessed by fluorescence in-situ hybridization (FISH) or chromogenic in-situ hybridization (CISH). Patients with such cancers may benefit from trastuzumab therapy, either as a single agent or in combination with chemotherapy. In metastatic breast cancer (MBC), objective responses to first-line singleagent trastuzumab were seen in $26 \%$ [9], but are substantially lower in patients who had received prior chemotherapy ( 12-15\%) [10,11]. Improved efficacy has been clearly demonstrated by the concurrent administration of trastuzumab with various chemotherapeutic agents [12-14]. In the pivotal MBC trial examining chemotherapy (anthracycline combined with cyclophosphamide, or paclitaxel) with or without trastuzumab, the addition of trastuzumab improved response rates, time to progression, and overall survival when compared to chemotherapy alone [12].

\section{Trastuzumab-related side-effects}

Trastuzumab is generally well-tolerated, and immediate reactions such as infusion-related hypersensitivity are relatively rare. Trastuzumab-associated cardiotoxicity, however, is the primary safety concern for patients and clinicians [15]. Most commonly, this presents as an asymptomatic decrease in cardiac ejection fraction and, less frequently, as overt congestive heart failure (CHF). Type II chemotherapy-related cardiac dysfunction (CRCD), as trastuzumabassociated cardiotoxicity has been recently named [16], differs from typical anthracycline-induced cardiotoxicity (type I CRCD) in a variety of ways. Type II CRCD does not occur in all individuals exposed to the trastuzumab, it does not appear to be dose-related, and when present, is variable in its severity. Type II CRCD has no identifiable cardiac ultrastructural abnormalities, unlike those seen in patients exposed to prior anthracyclines [17]. The greatest distinction in CRCD is that type II appears to be largely reversible, whereas type I is not. Standard medical management of trastuzumab-associated CHF results in significant symptom improvement, with the vast majority $(\sim 80 \%)$ achieving long-term control [18].

The mechanism of type II CRCD involves, at least in part, the HER-2 pathway. Binding of HER-2 ECD appears to block essential HER-2 signaling required for the growth, repair, and survival of cardiomyocytes [19]. In the absence of any prior anthracycline exposure, the reported CRCD rate for trastuzumab is $3 \%$ (2\% New York Heart Association (NYHA) Class III/IV severity). The rate increases to 5\% (4\% NYHA Class III/IV) in anthracycline pre-treated patients, and likely reflects anthracycline-associated myocardial damage that reduces available baseline cardiac reserve, to compensate for the sequential CRCD effect of trastuzumab [18]. CRCD increases substantially with concurrent administration of trastuzumab and anthracycline, with a reported CRCD rate of $27 \%(16 \%$ NYHA Class III/IV) [12].

\section{The role of platinum-based chemotherapy in breast cancer}

The platinum coordination complex cis-diamminedichloroplatinum(II) (cisplatin) is the prototype metallochemotherapeutic [20]. One hundred and twentyfive years following the original synthesis and characterization of cisplatin, Rosenberg et al. reported that electrochemical reactions on platinum electrodes produced cisplatin and its corresponding tetrachloroplatinum(IV) analog, suppressing bacterial growth [21]. Pre-clinical experiments demonstrated anticancer effects of cisplatin [22] mediated by interactions with DNA to form intrastrand adducts and interstrand cross-links [23]. Platinum coordination complexes can also bind to other macromolecules such as RNA and intracellular proteins to further disrupt normal cellular mechanisms [24]. Clinical trials have since established cisplatin in lung, ovarian, esophageal, cervical, head and neck and germ cell cancers.

Like other traditional chemotherapeutic agents, cisplatin has untoward side-effects (nephrotoxicity, ototoxicity, and neuropathy) and clinical drug resistance does occur. These problems spurred the development 
of alternative platinum analogs [25]. The first cisplatin analog to be developed was carboplatin. It differs in both its molecular structure and also its side-effect profile: carboplatin produces a lower incidence of nephrotoxicity and ototoxicity because of its slower rate of conversion to active platinum aqueous species [26], but triggers more profound neutropenia and thrombocytopenia. Unfortunately, carboplatin shows a marked degree of cross-resistance when compared to cisplatin. The only platinum analog in current use that demonstrates incomplete cross-resistance is trans-L-1,2-diaminocyclohexaneoxalato platinum(II), more commonly referred to as oxaliplatin [27]. A fourth marketed platinum drug nedaplatin (cis-diammineglycoloato platinum (II)) has not yet shown clear advantages over cisplatin or carboplatin. Given the important activity of platinum-based therapeutics, other platinum compounds continue to be evaluated.

As single agents for advanced breast cancer, firstline response rates range from $47 \%$ to $54 \%$ with cisplatin $[28,29]$ and $20 \%$ to $50 \%$ with carboplatin. These single-agent rates diminish substantially in previously treated patients with an average response rate of $8.5 \%$ and $6 \%$, respectively [30]. In pre-treated MBC, one study showed single-agent oxaliplatin to have a response rate of $21 \%$ [31]. Higher response rates are seen when platinum is combined with other antineoplatic agents, particularly taxanes [32]. Despite demonstrated activity in MBC, the use of platinumbased chemotherapy in clinical practice has been hampered by the lack of proven survival effects, platinum-associated toxicity profiles, and the ready availability of other active chemotherapeutic agents in the treatment of the disease $[33,34]$. Recent studies suggesting synergistic interactions with trastuzumab have renewed interest in platinum-based breast cancer therapy.

\section{Pre-clinical rationale for platinum-based therapy in HER-2-positive breast cancer}

Pre-clinical experiments [35-38] have shown that trastuzumab potentiates the cytotoxicity of cisplatin. This effect is mediated by trastuzumab suppressing HER-2-associated signal transduction and, through unknown intermediates, inhibiting the repair of platinum-DNA complexes. This augmentation of platinum-induced DNA damage is restricted to cells expressing HER-2, thereby selectively producing a synergistic cytotoxic effect only in those cells with the HER-2 alteration [39,40].

In an comprehensive study with HER-2 overexpressing cell lines, Pegram and colleagues [41] demonstrated synergistic cell killing with trastuzumab in combination with docetaxel, 4-hydroxycyclophosphamide, vinorelbine, and carboplatin. The greatest synergistic interaction was reported for the triplet combination of trastuzumab, carboplatin, and docetaxel. Similarly, the triplet combinations of trastuzumab, gemcitabine and cisplatin, and trastuzumab, gemcitabine and carboplatin also produce synergistic effects compared to gemcitabine and trastuzumab alone [42].

\section{Platinum-trastuzumab combinations in $\mathrm{MBC}$}

The clinical experience with platinum-trastuzumab combination therapy in the HER-2 over-expressing $\mathrm{MBC}$ setting is consistent with the pre-clinical models. The first study to explore cisplatin with trastuzumab was an open-label, multicentre study in extensively pre-treated MBC. Women received a loading dose of trastuzumab (250 mg intravenously (i.v.)) on day 0 , followed by weekly doses of $100 \mathrm{mg}$ i.v. for 9 weeks, with concurrent cisplatin $\left(75 \mathrm{mg} / \mathrm{m}^{2}\right.$ i.v. $)$ on days 1,29 , and 57 . Of the 37 patients assessable for response, nine $(24.3 \%)$ achieved a progesterone receptor, nine (24.3\%) had a minor response or stable disease, and disease progression occurred in 19 (51.3\%). The median response duration was 5.3 months (range 1.6-18). Grade III/IV toxicity was observed in 22 of 39 patients (56\%). The toxicity profile mirrored that of cisplatin alone with the most common toxicities being cytopenias $(n=10)$, nausea/vomiting $(n=9)$, and asthenia $(n=5)$. The pharmacokinetics of trastuzumab were unaltered by coadministration of cisplatin [43].

The results of two phase II trials using the triplet combination of docetaxel, trastuzumab, and platinum (either cisplatin or carboplatin) were recently published [44]. The University of California at Los AngelesOncology Research Network (UCLA-ORN) and the Breast Cancer International Research Group (BCIRG) each enrolled 62 patients with HER-2-over-expressing breast cancer into their respective trials. Patients received a median of six cycles of docetaxel at $75 \mathrm{mg} / \mathrm{m}^{2}$, cisplatin at $75 \mathrm{mg} / \mathrm{m}^{2}$ (BCIRG 101 study) or carboplatin at area under the curve (AUC) $=6 \mathrm{mg} /$ ml-min (UCLA-ORN study) given on day 1 and then repeated every 21 days. Trastuzumab was given on day 1 , cycle $1(4 \mathrm{mg} / \mathrm{kg})$ and then continued weekly at $2 \mathrm{mg} / \mathrm{kg}$ until disease progression. Patient characteristics were comparable between trials with the exception that the UCLA-ORN patients were more heavily pre-treated, with $15 \%$ of the patients receiving prior adjuvant taxane therapy. In BCIRG 101, patients may have received prior adjuvant or neoadjuvant therapy, but no prior chemotherapy for advanced disease and no prior taxanes were allowed. Both regimens were well tolerated, with manageable toxicities. More frequent hematologic toxicities were observed 
Table 1. Randomized studies employing platinum and trastuzumab.

\begin{tabular}{lllll}
\hline Trial & Sample size & Patient population & Randomization & Status \\
\hline Robert study & 194 & MBC & PH & $\begin{array}{c}\text { Closed to accrual; interim } \\
\text { results reported }\end{array}$ \\
& & $\begin{array}{l}\text { HER-2-positive } \\
\text { (IHC 2+, 3+, or FISH+) }\end{array}$ & PCarboH & $\begin{array}{c}\text { Closed to accrual; interim } \\
\text { results reported }\end{array}$ \\
BCIRG 006 & 3204 & $\begin{array}{l}\text { Node-positive and high-risk } \\
\text { HER-2-positive (FISH) }\end{array}$ & AC $\times 4 \rightarrow \mathrm{T} \times 4$ & $\begin{array}{l}\text { AC } \times 4 \rightarrow \mathrm{T} \times 4+\mathrm{H} \times 1 \text { year } \\
\text { TCarboH } \times 6+\mathrm{H} \times 1 \text { year }\end{array}$ \\
BCIRG 007 & 263 & $\begin{array}{l}\text { TH } \\
\text { TCarboH }\end{array}$ & Closed to accrual \\
\hline
\end{tabular}

A: doxorubicin; C: cyclophosphamide; H: trastuzumab; P: paclitaxel; T: docetaxel.

in the UCLA-ORN (TCarboH) study than in patients in the BCIRG 101 study (TCisH), while more nonhematologic toxicities were seen in the BCIRG 101 study. One patient in each study developed reversible CHF. An overall response rate (ORR) of 79\% (49/62 patients) (95\% confidence interval $(\mathrm{Cl})=66-89 \%)$ was seen in the BCIRG 101 study and 58\% (34/59 evaluable patients) in the UCLA-ORN study $(95 \%$ $\mathrm{Cl}=44-70 \%)$. Median times to progression were 9.9 months $(95 \% \mathrm{Cl}=8.3-13.1$ months $)$ and 12.7 months $(95 \% \mathrm{Cl}=8.6-15.5$ months $)$ for patients in the BCIRG 101 and UCLA-ORN studies, respectively. ORR were higher and median time to progression was longer in the subset of patients whose tumors harbored the HER-2 gene amplification.

One phase III platinum-trastuzumab combination trial [45] was reported in abstract form, and updated in the accompanying oral presentation. In this firstline study in HER-2-over-expressing $(\mathrm{IHC} 2+$ and $3+)$ MBC, Robert et al. randomized 194 women to either paclitaxel with trastuzumab (PT) or paclitaxel with carboplatin and trastuzumab (PCarboT). Forty per cent of patients had received prior adjuvant chemotherapy. Paclitaxel was administered i.v. $175 \mathrm{mg} / \mathrm{m}^{2}$ and carboplatin at an AUC of 6 every 3 weeks. Trastuzumab was given in the standard weekly fashion. In the 160 evaluable patients, an ORR of $57 \%$ was seen with PCarboT and an ORR of $38 \%$ was reported for PT, and responses increased to $67 \%$ and $37 \%$, respectively, in the HER-2 $3+$ subsets $(P<0.01)$. Time to progression was also prolonged in the PCarboT group compared to PT (13 months vs. 7 months, $P=0.002$ ) and again, longer when only HER-2 $3+$ patients were included (17 months vs. 9 months, $P=0.004$ ). No unexpected toxicities were observed. Higher-grade III/IV hematologic toxicities were reported in the PCarboT group over that in the PT group, primarily neutropenia (54\% vs. $23 \%$ ) and thrombocytopenia $(8 \%$ vs. $1 \%)$. PCarboT also produced more nausea ( $5 \%$ vs. $1 \%$ ). No other differences were seen in neurologic toxicities, neutropenic fever, cardiac or pulmonary toxicity, and no toxic deaths occurred. Median overall survival had not yet been reached in the PCarboT group at the time of analysis, compared to 33.5 months in the PT arm.

Although the docetaxel-trastuzumab combination has pre-clinical synergy [41] and striking clinical activity [13], the incremental effect of adding carboplatin to this doublet is unknown. BCIRG 007 was designed to address this question, and is a multicenter phase III randomized trial comparing the safety and efficacy of docetaxel-trastuzumab combination to docetaxelcarboplatinum trastuzumab in patients with HER-2 amplified MBC (see Table 1). At present, the trial has completed accrual with 263 patients, 185 patients have experienced an event, and an event-driven protocol-specified efficacy and safety analysis is expected shortly.

\section{Platinum-trastuzumab combination therapy: neoadjuvant setting}

The neoadjuvant setting is increasingly being used to evaluate the relative efficacy of novel systemic therapies, under the assumption that the short-term efficacy signals from pathologic responses will correlate with long-term survival outcomes. In a randomized neoadjuvant study in 42 women with HER-2-positive disease, the addition of trastuzumab to four cycles of paclitaxel followed by four cycles fluorouracil, epirubicin and cyclophosphamide, epirubicin markedly increased pathologic complete response rates $(65 \%$ vs. $26 \%, P=0.02$ ). Although clinical CHF was not seen in the 23 patients who received trastuzumab, safety outcomes and overall survival effects will remain uncertain due to the sample size [46].

A single trial of platinum-trastuzumab containing neoadjuvant chemotherapy is reported in abstract form [47]. Thirty-four patients with locally advanced breast cancer that over-expressed HER-2 $(2+$ or $3+$ by $\mathrm{IHC}$ ) were treated pre-operatively with standard schedule trastuzumab for 12 weeks, in combination 
with docetaxel $70 \mathrm{mg} / \mathrm{m}^{2}$ and cisplatin $70 \mathrm{mg} / \mathrm{m}^{2}$ on day $1,22,43$, and 64 , and G-CSF support. Patients then received four cycles of standard doxorubicin with cyclophosphamide (AC) therapy, surgery, followed by adjuvant radiotherapy with or without tamoxifen. Pathologic assessment of the breast revealed no invasive tumor in $21 \%(6 / 28)$, and axillary nodes were negative in $54 \%(15 / 28)$. The most common toxicities were alopecia (94\%), nausea/vomiting $(79 \%)$, asthenia (76\%), hyperglycemia (69\%), and anemia (61\%). Grade III toxicities included hyperglycemia (33\%), nausea/vomiting (6\%), and catheter infection (13\%). A decrease in left ventricular ejection fraction (LVEF) was noted in $45 \%(12 / 27)$ patients who had completed neoadjuvant therapy, possibly due to an interaction between residual circulating trastuzumab and doxorubicin. Given the uncertain long-term outcomes of this population, and the high rates of cardiotoxicity, this regimen of docetaxel+cisplatin + trastuzumab followed by doxorubicin with cyclophosphamide cannot be recommended.

\section{Platinum-trastuzumab combination therapy: adjuvant setting}

Four large scale, randomized phase III studies are evaluating the role of trastuzumab in the adjuvant setting. Preliminary efficacy and safety data has been reported for three of these trials $[48,49]$. The fourth trial, in which a platinum-trastuzumab combination is studied, has reported substantial cardiac safety information in an oral presentation [50], while some efficacy data was recently issued in a press release [51].

The National Surgical Adjuvant Breast and Bowel Project-B31 (NSABP-B31) protocol randomized 1736 node-positive, HER-2-positive breast cancer patients to four cycles of standard AC followed by four cycles of paclitaxel (every 3 weeks) with or without weekly trastuzumab ( $\times 1$ year). The Intergroup Protocol N9831 was a three-arm study that randomized 1615 patients following four cycles of AC chemotherapy to weekly paclitaxel ( $\times 12$ weeks) with or without weekly trastuzumab $(\times 1$ year) and allows for comparison of concurrent (with paclitaxel) vs. sequential trastuzumab therapy. With a combined median follow-up of 2.0 years (2.4 years in the NSABP-B31 and 1.5 years in the Intergroup trial) the results of a non-protocol (but Food and Drug Administration (FDA) approved) combined analysis showed significant benefits in those patients receiving adjuvant trastuzumab. The primary endpoint of disease-free survival (DFS) was improved in the trastuzumab containing arms ( $87 \%$ vs. $75 \% 3$ years DFS, hazard ratio (HR) 0.48 , $P=3 \times 10^{-12}$ ), and remained so in all subsets. Preliminary data also indicated longer overall survival in trastuzumab-treated patients as well (HR 0.67, $2 P=0.015)$. Trastuzumab therapy was, unfortunately, also associated with a significant increase in cardiotoxicity, increasing absolute rates of clinical CHF by $3.5 \%$. Data from the NSABP-B31 study suggests that $\sim 20 \%$ of patients discontinued trastuzumab due to either symptomatic or asymptomatic cardiotoxicity, with increasing age and post-AC chemotherapy LVEF being the main predictors for developing CHF. The trastuzumab-related fall in LVEF was largely reversible, with $\sim 68 \%$ of patients having symptoms resolve within 6 months.

The Herceptin Adjuvant Trial (HERA) is an international clinical trial led by the Breast International Group (BIG) in which over 5000 HER-2+ patients were randomized into one of three arms following the completion of adjuvant/ neoadjuvant chemotherapy. Patients received either the standard (no further treatment) or trastuzumab (administered every 3 weeks). Those receiving trastuzumab were randomly assigned to either 1 or 2 years of therapy. Efficacy results of the standard and 1-year trastuzumab therapy treatment arms were reported. With a median follow-up of only 1 year, improvement in the primary endpoint of DFS was seen in those who received trastuzumab (2 years DFS $85.8 \%$ vs. $77.4 \%$, HR $0.54,95 \% \mathrm{Cl}$ $0.43-0.67, P<0.0001)$. The risk of grade III/IV CHF was reported to be $0 \%$ in the observation arm and $0.5 \%$ with trastuzumab treatment.

The final adjuvant trastuzumab trial, Breast Cancer International Research Group 006 (BCIRG 006) is the only adjuvant study to address a trastuzumabplatinum question. This protocol randomized 3222 women with HER-2 amplified, node-positive or highrisk node-negative, operable breast cancer to one of three arms: (1) four cycles of standard AC chemotherapy followed by four cycles of docetaxel, (2) four cycles of standard AC chemotherapy, followed by four cycles of docetaxel with concurrent weekly trastuzumab then every three weekly trastuzumab to complete 1 year of antibody therapy, and (3) the docetaxelcarboplatin-trastuzumab regimen for six cycles, followed by weekly trastuzumab to complete 1 year of the antibody (Table 1). In a cardiac safety assessment release for publication by the independent cardiac evaluation panel, grade III/IV cardiac events were highest seen at rates of $1.2 \%, 2.3 \%$, and $1.2 \%$ in the three arms, respectively. Grade III/IV clinical CHF was reported in a single patient on arm 1, 18 patients in arm 2, and one patient in arm 3 [50]. A protocolspecified, event-driven interim efficacy analysis showed that adding trastuzumab to docetaxel following AC chemotherapy or adding trastuzumab to docetaxel and carboplatin chemotherapies improved DFS compared to chemotherapy alone. The reduction in the risk of disease recurrence, the primary endpoint 
of the study, was $51 \%(95 \% \mathrm{Cl}$ of $35-63 \%)$ in arm 2, and $39 \%(95 \% \mathrm{Cl}$ of $21-53 \%)$ in the docetaxelcarboplatin-trastuzumab third arm. The two experimental arms do not significantly differ in efficacy, and further follow-up will be required to determine the relative efficacy of the two trastuzumab-based regimens [51]. An updated cardiac safety assessment, full toxicity, and efficacy data will be reported at the 2005 San Antonio Breast Cancer Symposium.

\section{Conclusion}

The story of trastuzumab-platinum combination therapy highlights the challenges and the opportunities presented by new targeted breast cancer therapies. With a growing understanding of breast cancer biology, we now have the opportunity to evaluate targeted combinations in pre-clinical models, select appropriate patients for treatment, and choose the most promising targeted combinations to test in the 'clinical laboratory'. Although not yet complete, this story does provide one of the few examples of rational drug development and non-empiric trial design that has provided new, effective treatment options for women with breast cancer.

\section{References}

1. Parkin DM, Bray F, Ferlay J, Pisani P. Global cancer statistics, 2002. CA Cancer J Clin 2005; 55: 74-108.

2. Cancer Facts and Figures 2005. American Cancer Society, Inc., 2005. (Accessed 2005, 2005, at http://www. cancer.org/docroot/STT/content/STT_1x_Cancer_Facts_ Figures_2005.asp.)

3. King CR, Kraus MH, Aaronson SA. Amplification of a novel v-erbB-related gene in a human mammary carcinoma. Science 1985; 229: 974-976.

4. Slamon DJ, Clark GM, Wong SG, Levin WJ, Ullrich A, McGuire WL. Human breast cancer: correlation of relapse and survival with amplification of the HER-2/neu oncogene. Science 1987; 235: 177-182.

5. Stern DF, Heffernan PA, Weinberg RA. p185, a product of the neu proto-oncogene, is a receptorlike protein associated with tyrosine kinase activity. Mol Cell Biol 1986; 6: 1729-1740.

6. Masood S, Bui MM. Prognostic and predictive value of HER2/neu oncogene in breast cancer. Microsc Res Tech 2002; 59: 102-108.

7. Piccart M, Lohrisch C, Di Leo A, Larsimont D. The predictive value of HER2 in breast cancer. Oncology 2001; 61(Suppl 2): 73-82.

8. Nahta R, Esteva FJ. HER-2-targeted therapy: lessons learned and future directions. Clin Cancer Res 2003; 9: 5078-5084.

9. Vogel CL, Cobleigh MA, Tripathy D, et al. Efficacy and safety of trastuzumab as a single agent in first-line treatment of HER2-overexpressing metastatic breast cancer. J Clin Oncol 2002; 20: 719-726.

10. Baselga J, Tripathy D, Mendelsohn J, et al. Phase II study of weekly intravenous recombinant humanized
anti-p185HER2 monoclonal antibody in patients with HER2/neu-overexpressing metastatic breast cancer. J Clin Oncol 1996; 14: 737-744.

11. Cobleigh MA, Vogel CL, Tripathy $D$, et al. Multinational study of the efficacy and safety of humanized anti-HER2 monoclonal antibody in women who have HER2-overexpressing metastatic breast cancer that has progressed after chemotherapy for metastatic disease. J Clin Oncol 1999; 17: 2639-2648.

12. Slamon DJ, Leyland-Jones B, Shak S, et al. Use of chemotherapy plus a monoclonal antibody against HER2 for metastatic breast cancer that overexpresses HER2. New Engl J Med 2001; 344: 783-792.

13. Marty M, Cognetti F, Maraninchi D, et al. Randomized phase II trial of the efficacy and safety of trastuzumab combined with docetaxel in patients with human epidermal growth factor receptor 2-positive metastatic breast cancer administered as first-line treatment: the M77001 study group. J Clin Oncol 2005; 23: 4265-4274.

14. Burstein HJ, Harris LN, Marcom PK, et al. Trastuzumab and vinorelbine as first-line therapy for HER2-overexpressing metastatic breast cancer: multicenter phase II trial with clinical outcomes, analysis of serum tumor markers as predictive factors, and cardiac surveillance algorithm. J Clin Oncol 2003; 21: 2889-2895.

15. Perez EA, Rodeheffer R. Clinical cardiac tolerability of trastuzumab. J Clin Oncol 2004; 22: 322-329.

16. Ewer MS, Lippman SM. Type II chemotherapy-related cardiac dysfunction: time to recognize a new entity. J Clin Oncol 2005; 23: 2900-2902.

17. Minotti G, Menna P, Salvatorelli E, Cairo G, Gianni L. Anthracyclines: molecular advances and pharmacologic developments in antitumor activity and cardiotoxicity. Pharmacol Rev 2004; 56: 185-229.

18. Seidman A, Hudis C, Pierri MK, et al. Cardiac dysfunction in the trastuzumab clinical trials experience. $J$ Clin Oncol 2002; 20: 1215-1221.

19. Negro A, Brar BK, Lee KF. Essential roles of Her2/erbB2 in cardiac development and function. Recent Prog Horm Res 2004; 59: 1-12.

20. Fuertes MA, Alonso C, Perez JM. Biochemical modulation of cisplatin mechanisms of action: enhancement of antitumor activity and circumvention of drug resistance. Chem Rev 2003; 103: 645-662.

21. Rosenberg B, Vancamp L, Krigas T. Inhibition of cell division in Escherichia Coli by electrolysis products from a platinum electrode. Nature 1965; 205: 698-699.

22. Rosenberg B, VanCamp L, Trosko JE, Mansour VH. Platinum compounds: a new class of potent antitumour agents. Nature 1969; 222: 385-386.

23. Go RS, Adjei AA. Review of the comparative pharmacology and clinical activity of cisplatin and carboplatin. J Clin Oncol 1999; 17: 409-422.

24. Pascoe JM, Roberts JJ. Interactions between mammalian cell DNA and inorganic platinum compounds. I. DNA interstrand cross-linking and cytotoxic properties of platinum(II) compounds. Biochem Pharmacol 1974; 23: 1359-1365.

25. Wong E, Giandomenico CM. Current status of platinumbased antitumor drugs. Chem Rev 1999; 99: 2451-2466.

26. Judson I, Kelland LR. New developments and approaches in the platinum arena. Drugs 2000; 59(Suppl 4): 29-36.

27. Fuertes MA, Castilla J, Alonso C, Perez JM. Novel concepts in the development of platinum antitumor drugs. Curr Med Chem Anti-Canc Agents 2002; 2: 539-551. 
28. Sledge Jr GW, Loehrer Sr PJ, Roth BJ, Einhorn LH. Cisplatin as first-line therapy for metastatic breast cancer. J Clin Oncol 1988; 6: 1811-1814.

29. Kolaric K, Roth A. Phase II clinical trial of cis-dichlorodiammine platinum (cis-DDP) for antitumorigenic activity in previously untreated patients with metastatic breast cancer. Cancer Chemother Pharmacol 1983; 11: 108-112.

30. Decatris MP, Sundar S, O’Byrne KJ. Platinum-based chemotherapy in metastatic breast cancer: current status. Cancer Treat Rev 2004; 30: 53-81.

31. Garufi C, Nistico C, Brienza S, et al. Single-agent oxaliplatin in pretreated advanced breast cancer patients: a phase II study. Ann Oncol 2001; 12: 179-182.

32. Crown J, Pegram M. Platinum-taxane combinations in metastatic breast cancer: an evolving role in the era of molecularly targeted therapy. Breast Cancer Res Treat 2003; 79(Suppl 1): S11-S18.

33. Martin M. Platinum compounds in the treatment of advanced breast cancer. Clin Breast Cancer 2001; 2: 190-208.

34. Smith IE, Talbot DC. Cisplatin and its analogues in the treatment of advanced breast cancer: a review. $\mathrm{Br} J$ Cancer 1992; 65: 787-793.

35. Aboud-Pirak E, Hurwitz E, Pirak ME, Bellot F, Schlessinger J, Sela M. Efficacy of antibodies to epidermal growth factor receptor against $\mathrm{KB}$ carcinoma in vitro and in nude mice. J Natl Cancer Inst 1988; 80: 1605-1611.

36. Hancock MC, Langton BC, Chan T, et al. A monoclonal antibody against the c-erbB-2 protein enhances the cytotoxicity of cis-diamminedichloroplatinum against human breast and ovarian tumor cell lines. Cancer Res 1991; 51: 4575-4580.

37. Pietras RJ, Pegram MD, Finn RS, Maneval DA, Slamon DJ. Remission of human breast cancer xenografts on therapy with humanized monoclonal antibody to HER-2 receptor and DNA-reactive drugs. Oncogene 1998; 17: 2235-2249.

38. Pegram M, Hsu S, Lewis G, et al. Inhibitory effects of combinations of HER-2/neu antibody and chemotherapeutic agents used for treatment of human breast cancers. Oncogene 1999; 18: 2241-2251.

39. Pietras RJ, Fendly BM, Chazin VR, Pegram MD, Howell SB, Slamon DJ. Antibody to HER-2/neu receptor blocks DNA repair after cisplatin in human breast and ovarian cancer cells. Oncogene 1994; 9: 1829-1838.

40. Arteaga CL, Winnier AR, Poirier MC, et al. p185c-erbB-2 signal enhances cisplatin-induced cytotoxicity in human breast carcinoma cells: association between an oncogenic receptor tyrosine kinase and drug-induced DNA repair. Cancer Res 1994; 54: 3758-3765.

41. Pegram MD, Konecny GE, O'Callaghan C, Beryt M, Pietras R, Slamon DJ. Rational combinations of trastuzumab with chemotherapeutic drugs used in the treatment of breast cancer. J Natl Cancer Inst 2004; 96: 739-749.

42. Konecny GE, Pegram MD. Gemcitabine in combination with trastuzumab and/or platinum salts in breast cancer cells with HER2 overexpression. Oncology (Williston Park) 2004; 18(14 Suppl 12): 32-36.

43. Pegram MD, Lipton A, Hayes DF, et al. Phase II study of receptor-enhanced chemosensitivity using recombinant humanized anti-p185HER2/neu monoclonal antibody plus cisplatin in patients with HER2/neu-overexpressing metastatic breast cancer refractory to chemotherapy treatment. J Clin Oncol 1998; 16: 2659-2671.

44. Pegram MD, Pienkowski T, Northfelt DW, et al. Results of two open-label, multicenter phase II studies of docetaxel, platinum salts, and trastuzumab in HER2-positive advanced breast cancer. J Natl Cancer Inst 2004; 96: 759-769.

45. Robert N, Leyland-Jones B, Asmar L, et al. Phase III comparative study of trastuzumab and paclitaxel with and without carboplatin in patients with HER2/neu positive advanced breast cancer. Breast Cancer Res Treat 2002; 76 (Suppl 1): S37 [Abstract 35].

46. Buzdar AU, Ibrahim NK, Francis D, et al. Significantly higher pathologic complete remission rate after neoadjuvant therapy with trastuzumab, paclitaxel, and epirubicin chemotherapy: results of a randomized trial in human epidermal growth factor receptor 2-positive operable breast cancer. J Clin Oncol 2005; 23: 3676-3685.

47. Hurley JDP, Silva O, Gomez-Fernandez C, Reis I, Velez P, Castillo M, Bateman D, Franco S, Gautam U. Neoadjuvant herceptin/taxotere/cisplatin in the treatment of locally advanced and inflammatory breast cancer [Abstract 50]. In: Steven M. Grunberg (Ed.). Proceedings of the 38th Annual Meeting of the American Society of Clinical Oncology, Orlando, FL. Baltimore, MD, Lippincott Williams \& Wilkins, 18-21 May 2002.

48. Romond EPE, Bryant $\mathrm{J}$, et al. Adriamycin and cyclophosphamide followed by paclitaxel with or without trastuzumab as therapy in patients with HER2-positive operative breast cancer. Combined analysis of NSABP B-31 and NCCTG N9831 (oral presentation during symposium on Advances in Monoclonal Antibody Therapy for Breast Cancer). In: 41st Annual Meeting of the American Society of Clinical Oncology, Orlando, FL, 13-17 May 2005.

49. Piccart M. HERA trial (oral presentation during symposium on Advances in Monoclonal Antibody Therapy for Breast Cancer). In: 41st Annual Meeting of the American Society of Clinical Oncology, May 13-17, Orlando, FL, 2005.

50. Slamon D. ASCO Educational Session, Antibody-based therapeutics: more than a one-trick pony. HER-2/neu: target identification and target validation in pathogenesis clinical application. Orlando, FL, 2005.

51. Fourth Phase III Study Shows Herceptin Plus Chemotherapy Improved Disease-Free Survival in Early-Stage HER2-Positive Breast Cancer, 2005. (Accessed September 13, 2005, at http://www.gene.com/gene/news/pressreleases/display.do?method $=$ detail\&id $=8847$.) 